\title{
High fidelity imaging of geosynchronous satellites with the MROI
}

\author{
John Young ${ }^{\mathrm{a}}$, Christopher Haniffa ${ }^{\mathrm{a}}$, David Buscher ${ }^{\mathrm{a}}$, Michelle Creech-Eakman ${ }^{\mathrm{b}}$, and Ifan Payne ${ }^{\mathrm{b}}$ \\ ${ }^{a}$ Cavendish Laboratory, University of Cambridge, JJ Thomson Avenue, Cambridge CB3 OHE, UK \\ ${ }^{\mathrm{b}}$ Magdalena Ridge Observatory, New Mexico Institute of Mining and Technology, Socorro, NM \\ 87801, USA
}

\begin{abstract}
Interferometry currently provides the only practicable way to image satellites in Geosynchronous Earth Orbit (GEO) with sub-meter spatial resolution. The Magdalena Ridge Observatory Interferometer (MROI) is being funded by the US Air Force Research Laboratory to demonstrate the 9.5 magnitude sensitivity (at $2.2 \mu \mathrm{m}$ wavelength) and baseline-bootstrapping capability that will be needed to realize a useful turn-key GEO imaging capability. This program will utilize the central three telescopes of the MROI and will aim to validate routine acquisition of fringe data on faint well-resolved targets. In parallel with this effort, the University of Cambridge are investigating the spatial resolution and imaging fidelity that can be achieved with different numbers of array elements. We present preliminary simulations of snapshot GEO satellite imaging with the MROI. Our results indicate that faithful imaging of the main satellite components can be obtained with as few as 7 unit telescopes, and that increasing the number of telescopes to 10 improves the effective spatial resolution from 0.75 meter to 0.5 meter and enables imaging of more complex targets.
\end{abstract}

Keywords: optical interferometry, image reconstruction, geosynchronous satellite

\section{INTRODUCTION}

Current ground-based filled-aperture telescopes do not provide a useful capability for imaging satellites in Geosynchronous Earth Orbit (GEO). The only practicable way to image GEO satellites with sub-meter resolution is to employ visible/infrared interferometry. The Magdalena Ridge Observatory Interferometer (MROI) has secured funding from the US Air Force Research Laboratory to determine the feasibility of imaging passively-illuminated GEO satellites with a groundbased interferometer, by means of two parallel activities:

- A demonstration of the 9.5 mag. sensitivity and baseline-bootstrapping capability that will be needed to realize a useful GEO imaging capability. This program will utilize the central three telescopes of the MROI and aims to validate routine acquisition of fringe data on faint well-resolved targets.

- Computational simulations to investigate the spatial resolution and imaging fidelity that can be achieved with different numbers of array elements. The simulation framework will also be used to investigate other factors that can potentially limit the imaging performance, such as albedo variations with wavelength and varying solar illumination during the observations.

The MROI has been designed as an ambitious visible/near-infrared synthesis array, optimized for fast and reliable imaging of faint and complex celestial targets. The design comprises an array of up to ten 1.4-meter diameter unit telescopes (UT) arranged in an equilateral "Y" configuration. ${ }^{1}$ Each of these UTs will collect light from a celestial source and send a collimated, stabilized beam of light to a central beam combining laboratory. There, the beams will be path-equalized and superposed to generate interference fringes that encode information about the brightness distribution of the source, at spatial frequencies corresponding to the vector separations between the unit telescopes.

The MROI's unit telescopes will be re-locatable amongst a set of 28 separate foundation pads, so as to give intertelescope separations (i.e. baseline lengths) from 7.8 meters to 347 meters. At its shortest operating wavelength of $650 \mathrm{~nm}$, the MROI will have a maximum angular resolution of approximately 0.35 milliarcseconds. The more compact array configurations (the most closely packed one is shown in Fig. 1) will be matched to the angular scales subtended by satellites in Geosynchronous Earth Orbit (GEO) at near-infrared wavelengths (Fig. 2), allowing imaging at a resolution from $1 \mathrm{~m}$ down to $10 \mathrm{~cm}$. 


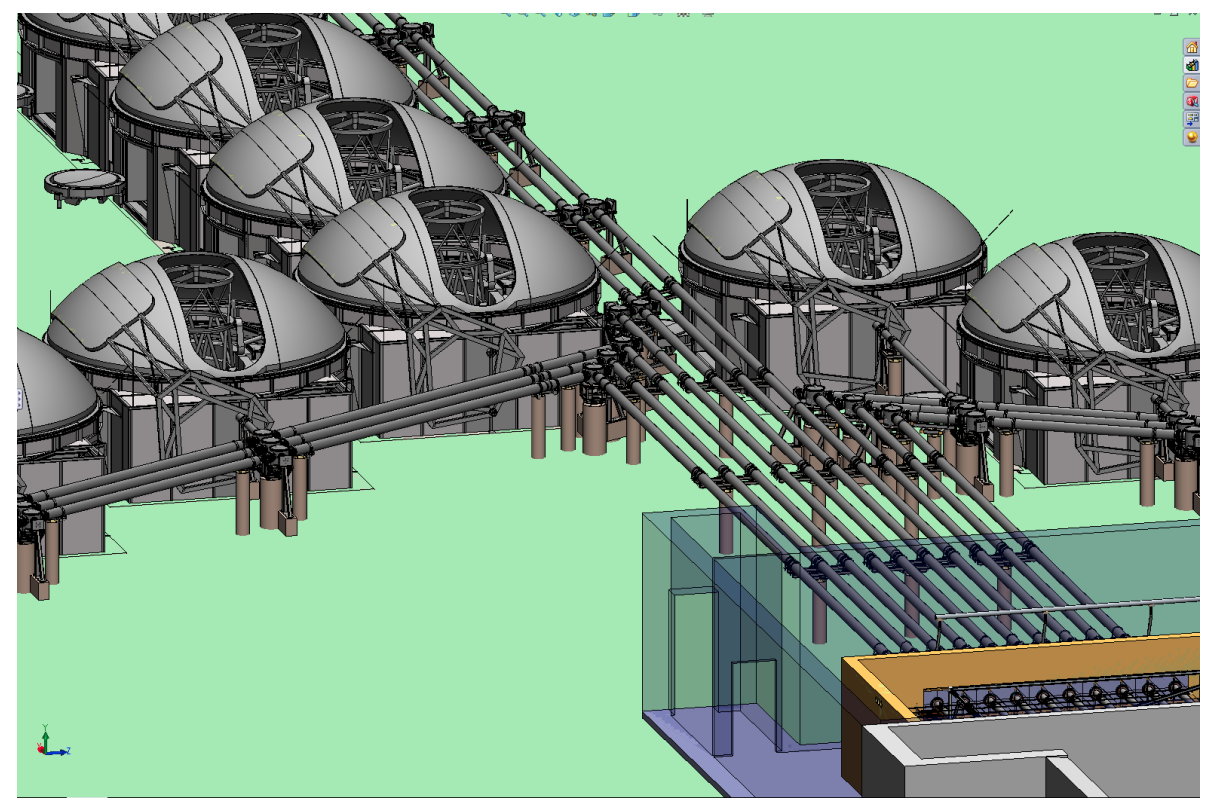

Figure 1: A rendering from a CAD model of the MROI array, showing the telescopes in a close-packed configuration with $7.8 \mathrm{~m}$ center-to-center spacings. Part of the beam combining facility is visible in the foreground.

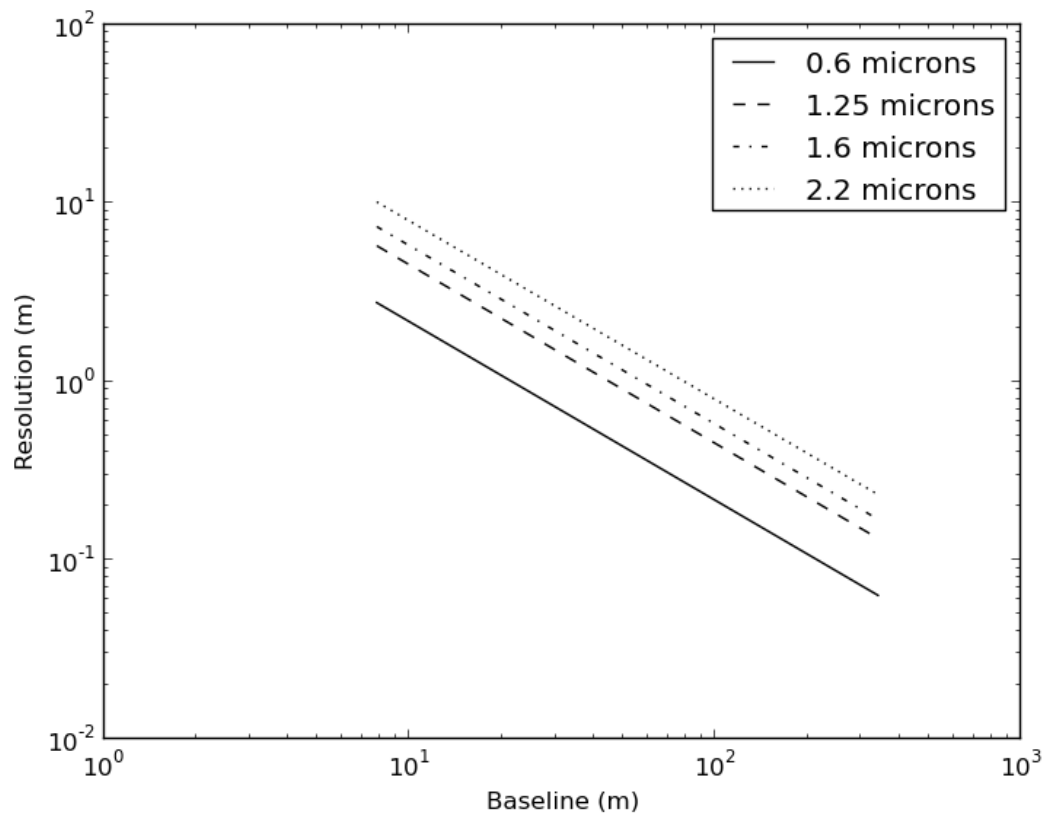

Figure 2: The relationship between the available MROI baseline lengths, the observing wavelength and the interferometric resolution (in meters) assuming a target in geosynchronous orbit. 


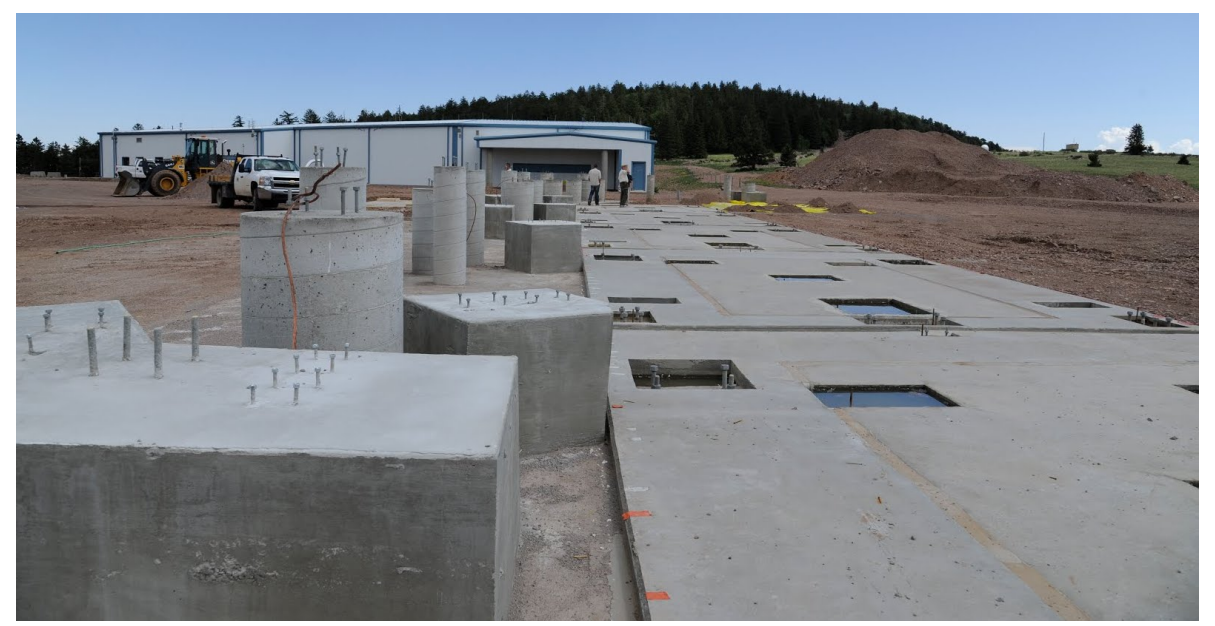

Figure 3: The first three foundation pads for unit telescopes on the West arm of the MROI, together with the beam-relay piers. A total of seven foundation pads have been constructed so far. The MROI beam combining facility is in the background.

The majority of the MROI sub-systems have passed their final design review, and where applicable, been tested in the laboratory. The remaining technical risks pertaining to satellite imaging are associated with meeting the sensitivity goals for the array and successfully integrating the component parts into an instrument that works efficiently and reliably. These risks can only be retired by commissioning the sub-systems on the observatory site and carrying out an end-to-end demonstration of the interferometer's performance.

\section{SENSITIVITY DEMONSTRATION}

We have begun a five-year program to mitigate the primary technical risks associated with developing a ground-based satellite imaging array. Under this program, three unit telescopes will be installed on the Magdalena Ridge, and used to secure interferometric data on GEO targets. These measurements will be used to validate models of the instrumental performance needed to predict the imaging capability of the full telescope array.

The MROI sub-systems will be deployed and tested in sequence, starting with the unit telescopes, beam relay system, and delay lines. The demonstration will culminate in measurements of co-phased visibilities (year four) and closure phases (year five) for GEO targets.

At the time of writing, the first UT is in the process of being commissioned on the Magdalena Ridge following successful completion of its Factory Acceptance Tests. Initial tests of the telescope will be performed in a maintenance facility building on the Ridge prior to integration with the fast tip-tilt correction system and telescope enclosure. A short section of the beam relay system has been installed and is undergoing tests. The first delay line has been installed and initial tests of its performance have been carried out. Please refer to Ref. 2 for further information on the current status of the MROI and activities we plan to undertake.

Two major reviews of the on-sky system performance are planned during the five-year program. These reviews will utilize in-depth analysis of measured data in order to verify:

1. in year three: the stability in flux, tilt and shear of light delivered by a unit telescope after passing through the beam relay optics and delay line; and

2. in year five: baseline bootstrapping and closure phase measurement on faint targets using three telescopes. 

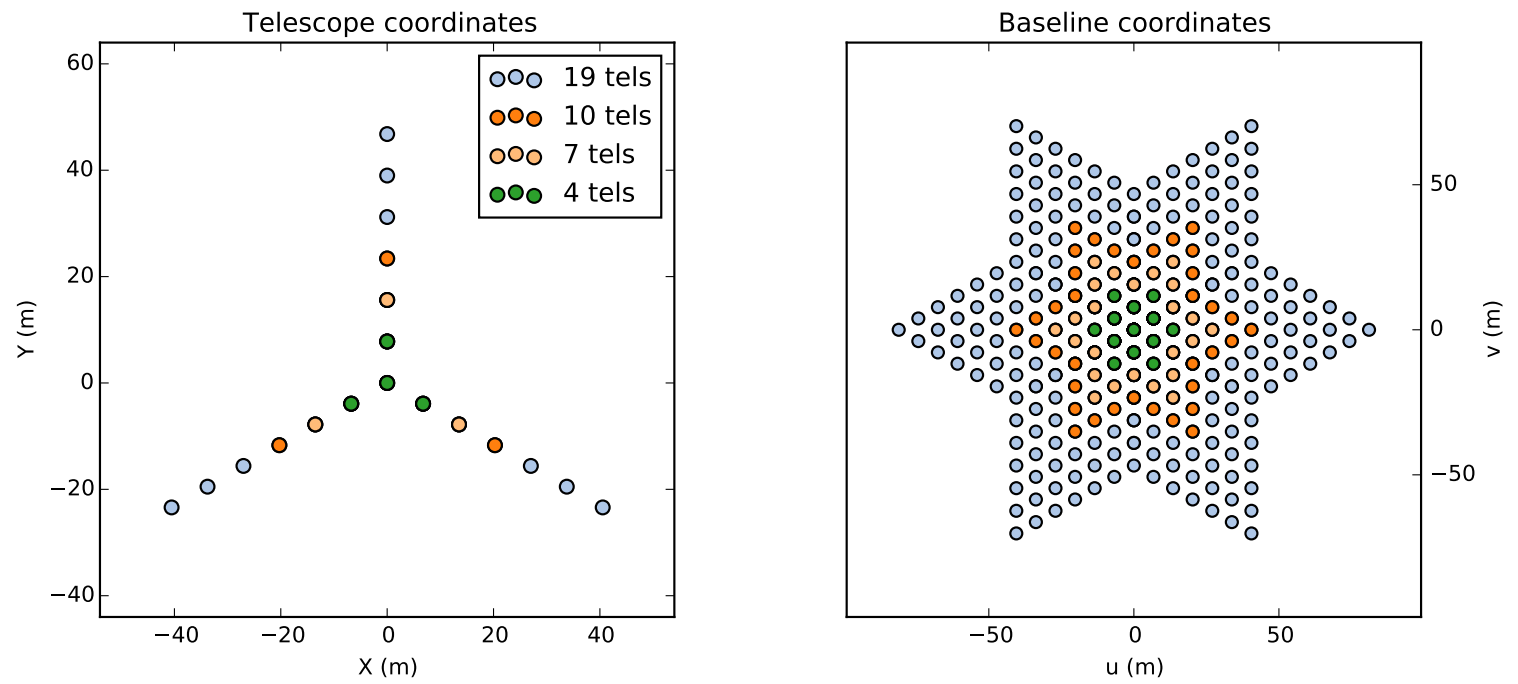

Figure 4: The telescope array layout for an MROI-like array design at different stages of development (left) and the $(u, v)$ plane coverage of these arrays (right), for a target at the zenith. Only the most compact configurations of these arrays are shown. The 19-telescope array would require some changes to the existing MROI infrastructure.

\section{IMAGING SIMULATIONS}

We have carried out preliminary simulations to demonstrate that imaging of a realistic satellite is possible using a modest number of apertures and the fringe signal-to-noise predicted for the MROI. These simulations will serve as the starting point for a more sophisticated analysis to be performed over the next year. This follow-on work will relax some of the simplifying assumptions used to obtain the results presented here, and investigate a range of satellite sizes and shapes.

Simulated interferometric datasets were prepared by evaluating the discrete Fourier transform of a suitably scaled model image at spatial frequencies corresponding to the projected MROI baselines. The assumed telescope array configurations and corresponding $(u, v)$ coordinates are shown in Fig. 4. The configurations were optimized for baseline bootstrapping, with a constant $7.8 \mathrm{~m}$ spacing between adjacent telescopes. The Fourier data were then used to generate the interferometric observables (squared visibilities and bispectra), with the addition of appropriate levels of measurement noise.

The MROI splits the light from the target by wavelength, separate wavebands being used for tip-tilt correction, fringe tracking, and "science" i.e. synthesis imaging of the target. For observations of brighter GEO targets, fringe tracker measurements will be used to co-phase the science fringe data, extending the coherent integration beyond the atmospheric coherence time and thus enhancing the signal-to-noise. For science observations at $K$ band (2.0-2.4 $\mu \mathrm{m}$ wavelength), which are needed to sample the low spatial frequency satellite structures, the best results will be obtained by sharing the $K$ band light between the fringe tracking and science combiners. These can be interleaved with $J$ band $(1.1-1.4 \mu \mathrm{m})$ observations to obtain complementary higher spatial frequency information. For targets fainter than 10th magnitude, the fringe tracker must be used in a "coherencing" mode and the integration time for the science combiner will then be limited to twice the atmospheric coherence time. The results presented here assume co-phased observations; simulations assuming coherencing were presented in Refs. 3,4.

The model image used for the simulations is shown in Fig. 8, and its visibility (ratio of coherent to total flux) as a function of projected baseline length for a $2.2 \mu \mathrm{m}$ observing wavelength is shown in Fig. 5. This spacecraft has structure on a range of scales from $\sim 0.2 \mathrm{~m}$ to $17 \mathrm{~m}$ and is therefore a good test of the accuracy and effective resolution with which images can be reconstructed. The assumed size is a good match to the most compact MROI configuration, as the short bootstrapping baselines sample the first lobe of the visibility function where the signal-to-noise is highest, and provide information on the overall size and shape of the satellite. Larger satellites will be brighter as they reflect more sunlight, but additional low spatial frequency information, for example from a nearby filled aperture telescope, will be needed to ensure successful image reconstruction. ${ }^{4}$ 


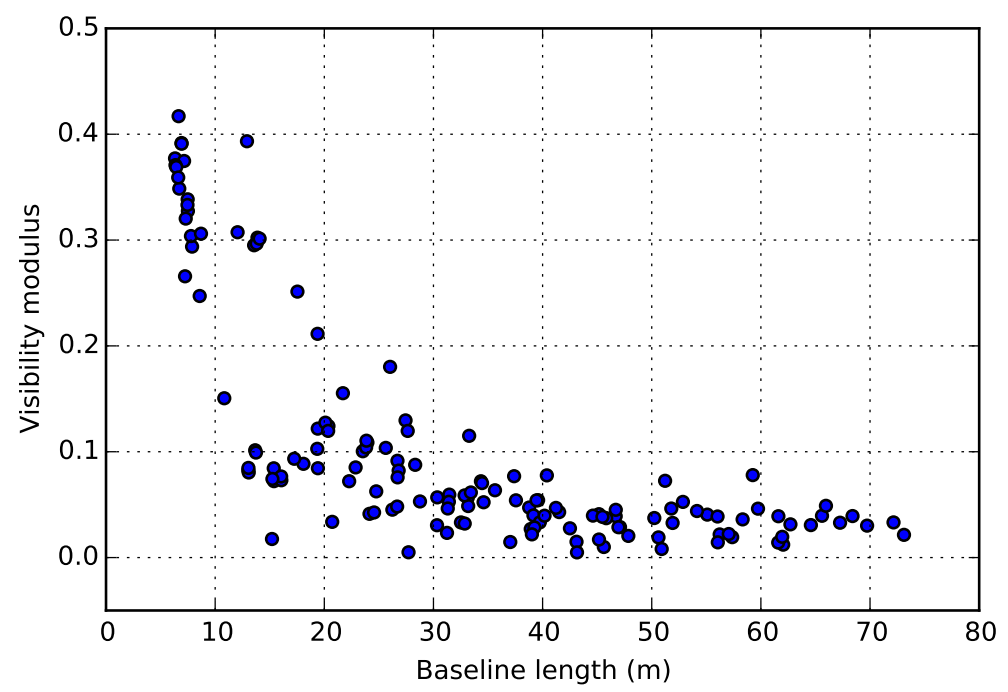

Figure 5: Object visibility as a function of baseline length at a wavelength of $2.2 \mu \mathrm{m}$ for the satellite shown in Fig. 8 , which has a "bus" of order $5 \mathrm{~m}$ in dimension and a $10 \mathrm{~m}$ long solar panel. Note the steep fall-off in visibility for baselines longer than about $8 \mathrm{~m}$.
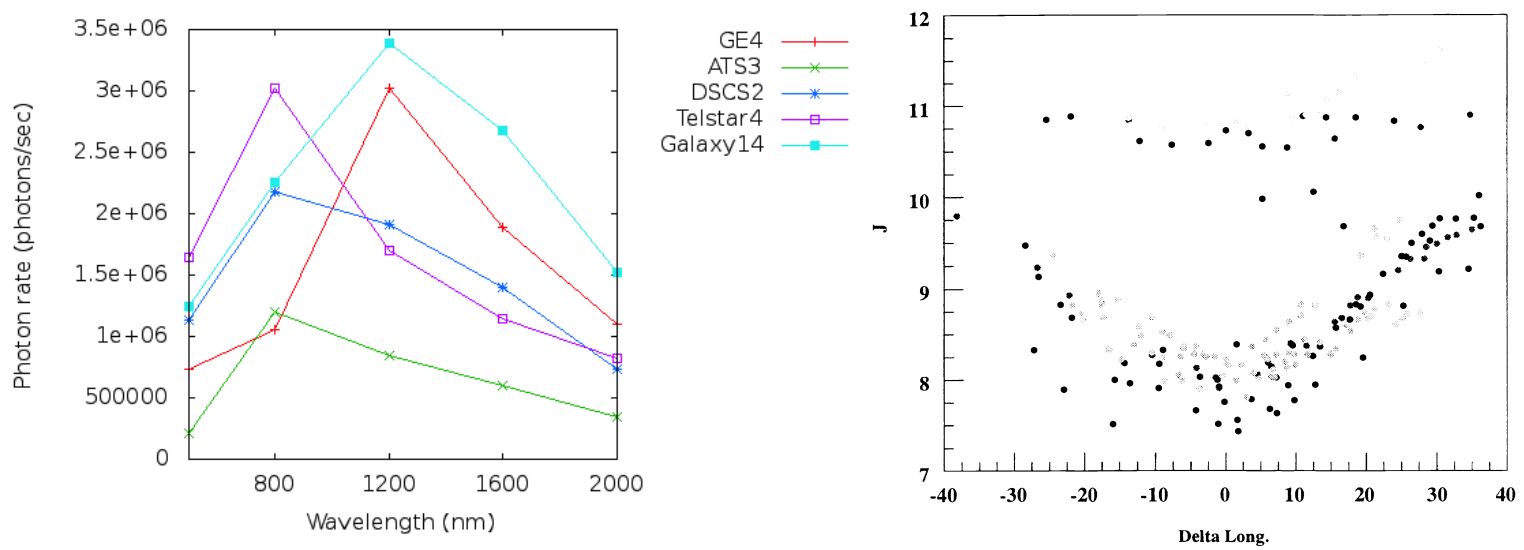

Figure 6: The photon flux in $400 \mathrm{~nm}$ bandpasses computed using TASAT software for a sample of GEO satellites (left) and $J$-band (1.2 $\mu \mathrm{m}$ wavelength) magnitudes measured ${ }^{5}$ on a range of satellites (right). The symbols in the right panel distinguish the various satellites observed (a different sample compared with the left panel).

The spacecraft's appearance was assumed to be identical in all of the observing wavebands. In reality, there will be variations in the albedos of the materials that form the spacecraft's surface with wavelength. These variations are most pronounced for the solar panels at visible wavelengths. We do not expect large albedo variations between the IR wavelengths considered here. The impact of any such variations will be explored in our future work.

Radiometric modeling of some typical GEO targets is shown on the left of Fig. 6. Converted to the astronomical magnitude system these targets have visible magnitudes in the range 10-13 and near-IR magnitudes in the range 8-11. Photometric measurements such as those shown on the right in Fig. 6 confirm this general range. We took the satellite to be 9.5 mag., in the middle of the expected brightness range. The assumed noise levels for the simulated data were based on realistic assumptions of co-phasing accuracy at this target brightness and the coherence losses predicted for the MROI optical train (Fig. 7). In addition, uncorrelated calibration errors were added to the visibilities $\left(\Delta V^{2} / V^{2}=0.02\right)$ and closure phases $\left(0.8^{\circ}\right)$.

We assumed the presence of a switchable science beam combiner which mixes 6 beams together simultaneously (using 192 pixels per spectral channel to sample the spatially-encoded fringes) and $4 \mathrm{e}^{-}$readout noise. Coherent and incoherent integration times of $10 \mathrm{~s}$ and $300 \mathrm{~s}$ respectively were assumed. For the ten-telescope simulation, the mean signal-to-noise 


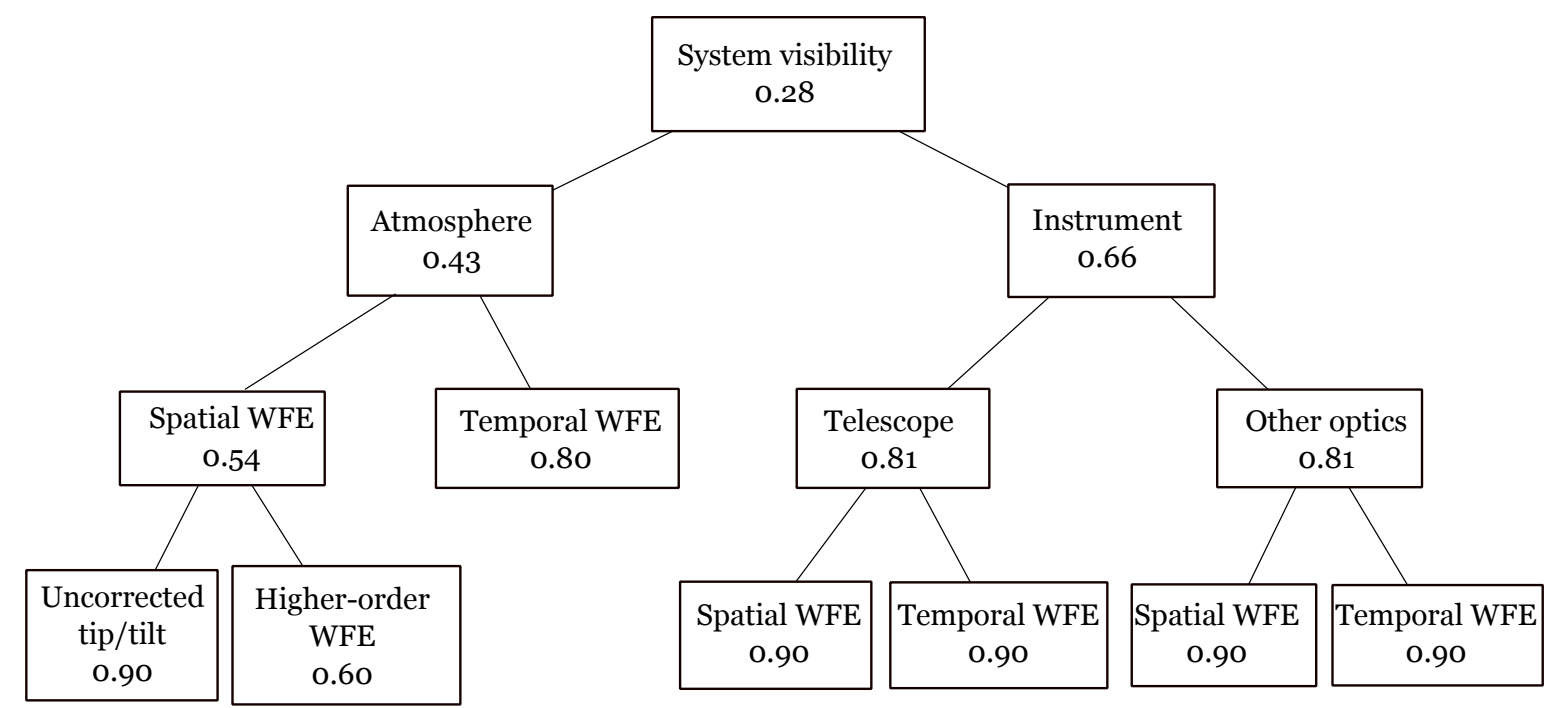

Figure 7: Schematic representation of the allocations for RMS fringe visibility loss from the MROI error budget. See Ref. 1 for more explanation.

ratio of the synthetic squared visibilities was 6.8 , and the mean closure phase error was $3.1^{\circ}$. The longest baselines had the largest errors (for example closure phase errors of $\sim 40^{\circ}$ on triangles of long baselines) as expected. The times taken to reconstruct an image were typically a few minutes on a standard desktop PC.

The simulated data were saved in the OIFITS format ${ }^{6}$ and used as input to the BSMEM image reconstruction code. ${ }^{7}$ For each dataset, a two-step reconstruction procedure was used. First, higher spatial frequency data were removed and BSMEM run with an uninformative circular Gaussian prior image. High spatial frequencies and noise were removed from the BSMEM output image by convolving with a circular Gaussian function and setting pixel values below a user-selected threshold to zero. The resulting image was used as a prior for a second run of BSMEM on the full dataset.

\section{SIMULATION RESULTS}

For arrays of 7 or more telescopes, the simulation results (Fig. 8) show faithful reconstructions of the spacecraft. The effective spatial resolution of the images increases as the number of telescopes, and hence the maximum baseline, is increased. These images exhibit "super-resolution" beyond the nominal $\lambda / B$ diffraction limit. For example, the $1 \mathrm{~m}$ diameter structure at the tip of the boom is resolved in the seven-telescope image where the nominal resolution is just $1.6 \mathrm{~m}$.

Observations using 4 telescopes provide information on the size and orientation of the satellite but, at least for such a moderately-complex target, do not permit model-independent imaging as only 6 spatial frequencies per wavelength channel are measured. As expected, BSMEM did not converge successfully on such datasets.

\section{CONCLUSIONS}

The MROI is being funded by the US Air Force Research Laboratory to prove the feasibility of imaging of passivelyilluminated GEO satellites from the ground using an interferometer. A three-telescope baseline-bootstrapping array is being deployed on the Magdalena Ridge, and will be used to demonstrate routine closure phase measurement on faint satellites and validate our models for the performance of the interferometer.

We have presented preliminary simulations of snapshot GEO satellite imaging with the MROI. Our results indicate that faithful imaging of the main satellite components can be obtained with as few as 7 unit telescopes, and that increasing the number of telescopes to 10 improves the effective spatial resolution from 0.75 meter to 0.5 meter and reveals more complex details in the reconstructed image.

Our future simulation work will consider a range of satellite sizes and shapes, the need for additional baselines shorter than $7.8 \mathrm{~m}$, and the impact of variations in the appearance of the satellite with time and wavelength. 

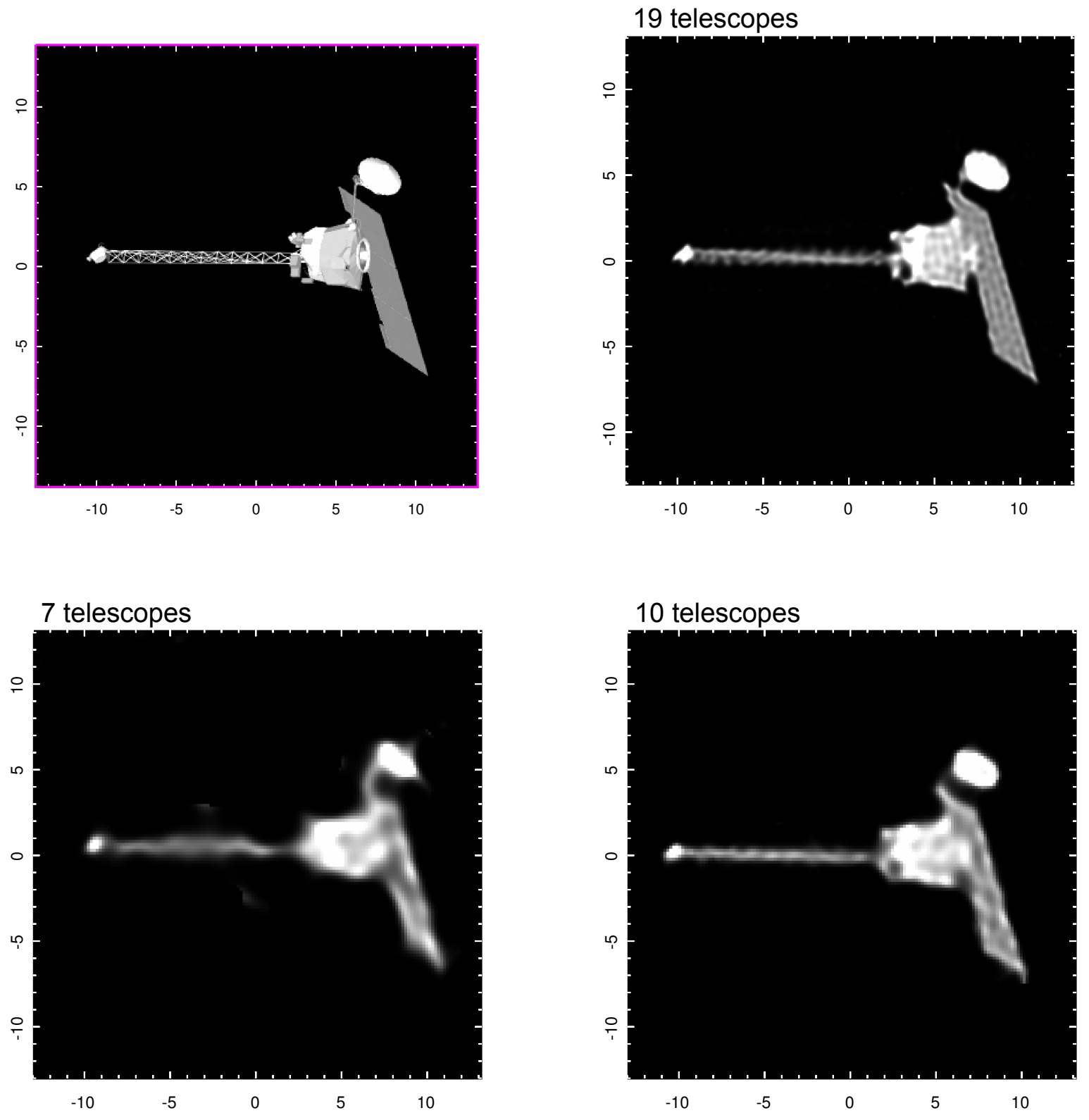

Figure 8: Truth (top left) and reconstructed images using 19, 10, and 7 telescopes (clockwise from top right) for a simulated MROI observation of a GEO satellite. The axes are scaled in meters. 


\section{REFERENCES}

[1] Buscher, D. F., Creech-Eakman, M. J., Farris, A., Haniff, C. A., and Young, J. S., "The Conceptual Design of the Magdalena Ridge Observatory Interferometer," Journal of Astronomical Instrumentation 2, 40001 (2013).

[2] Creech-Eakman, M. J., Payne, I., Romero, V. D., Haniff, C. A., Buscher, D. F., and Young, J. S., "A new path to first light for the Magdalena Ridge Observatory Interferometer," Proc. SPIE 9907 (2016). Paper 9907-4, these proceedings.

[3] Young, J. S., Haniff, C. A., Buscher, D. F., Creech-Eakman, M. J., Payne, I., Jurgenson, C. A., and Romero, V., "The MROI's capabilities for imaging geosynchronous satellites," Proc. SPIE 8445, 84452N (2012).

[4] Young, J. S., Haniff, C. A., and Buscher, D. F., "Interferometric imaging of geo-synchronous satellites with groundbased telescopes," in [Proceedings of the 2013 IEEE Aerospace Conference], 93 (2013).

[5] Sanchez, D. J., Gregory, S. A., Werling, D., Payne, T. E., Kann, L., Finkner, L. G., Payne, D. M., and Davis, C. K., "Photometric measurements of deep space satellites," Proc. SPIE 4091, 164 (2000).

[6] Pauls, T., Young, J., Cotton, W., and Monnier, J., "A data exchange standard for optical (visible/IR) interferometry," PASP 117, 1255 (2005).

[7] Buscher, D. F., "Direct maximum-entropy image reconstruction from the bispectrum," in [IAU Symp. 158: Very High Angular Resolution Imaging ], 91 (1994). 